\title{
COMPUTATION OF RELATIVISTIC SYMMETRY ORBITALS FOR FINITE DOUBLE POINT GROUPS
}

\author{
J. MEYER, W.-D. SEPP, B. FRICKE \\ Physics Department, University of Kassel, D-3500 Kassel, Fed. Rep. Germany
}

and

\begin{abstract}
A. ROSÉN
Physics Department, Chalmers University of Technology and University of Göteborg, S-41296 Göteborg, Sweden
\end{abstract}

Received 26 September 1988

A program is presented for the construction of relativistic symmetry-adapted molecular basis functions. It is applicable to 36 finite double point groups. The algorithm, based on the projection operator method, automatically generates linearly independent basis sets. Time reversal invariance is included in the program, leading to additional selection rules in the non-relativistic limit.

\section{PROGRAM SUMMARY}

Title of program: TSYM

Catalogue number: ABHW

Program obtainable from: CPC Program Library, Queen's University of Belfast, N. Ireland (see application form in this issue)

Computer: IBM 3090-200; Installation: Gesellschaft für Schwerionenforschung (GSI), Darmstadt, Fed. Rep. Germany

Operating system: MVS/XA

Programming language used: FORTRAN IV

Peripheral used: line printer (optional)

No. of bits in a word: 32

No. of lines in combined program and test deck: 6048
Keywords: molecule, relativistic symmetry orbital, time reversal, finite double point group

Nature of physical problem

Relativistic symmetry-adapted basis functions are generated for molecules of 36 finite point symmetry groups. Time reversal is included as an additional symmetry operation.

Method of solution

The symmetry orbitals are constructed by means of a projection operator technique. Linearly independent basis functions are selected.

Restrictions on the complexity of the program

The program is applicable to finite double point groups only. 36 groups are included.

Typical running time

Less than $30 \mathrm{~s}$. 


\section{LONG WRITE-UP}

\section{Introduction}

Group theory provides a valuable tool for the simplification of molecular calculations. By means of a projection operator formalism, symmetry-adapted molecular basis functions can be constructed from atomic orbitals. This method is widely used in quantum chemistry. It leads to a full exploitation of the spatial symmetry properties of the molecule and, as a consequence, greatly simplifies the diagonalization of the molecular single-electron Hamiltonian. The projection operator technique has been applied in relativistic molecular calculations by several authors, e.g. Malli and Oreg [1], Rosén [2], Pyykkö and Toivonen [3,4], Ellis and Goodman [5]. An additional symmetry property of the molecular Hamiltonian is its invariance under time reversal, leading to the twofold Kramers degeneracy in systems with half-integer angular momentum. The exploitation of time reversal in molecular calculations has been recently analysed by Hafner [6], Rösch [7], and Ellis and Goodman [5].

In a previous paper [8], we have presented a theory combining the exploitation of spatial and time reversal symmetry for finite double point groups. The influence of time reversal on molecular symmetry orbitals generated by means of projection operators is studied for two-component Pauli spinors. The results have been applied in a program computing relativistic molecular symmetry orbitals under consideration of time reversal. The structure of this program is described in the present paper. The algorithm is partly based on earlier calculations performed by Rosén [2]. In the version presented here, it has been extended to the 36 most important finite double point groups.

Moreover, the program contains an algorithm selecting out a set of linearly independent basis functions. In general, employing the projection operator technique will produce a number of linearly dependent symmetry orbitals. For calculations it is therefore necessary to select out the independent ones. A systematic method is developed in ref. [8].

The paper is divided into six sections. Section 2 contains a summary of the underlying theory. Details can be found in refs. [2,8]. Section 3 presents a survey of the program structure. The input data are described in section 4 and the output data in section 5. Finally, an example of the output is given in section 6 .

\section{Theory}

\subsection{Construction of symmetry orbitals}

The molecular wave functions are constructed as linear combinations of atomic orbitals (LCAO). The basis functions are Dirac four-spinors $[2,8]$

$$
|\rho a \kappa m\rangle:=\left(\begin{array}{c}
\frac{P_{n \kappa}\left(r_{a}\right)}{r_{a}} y_{\kappa m}\left(\vartheta_{a}, \varphi_{a}\right) \\
i \frac{Q_{n \kappa}\left(r_{a}\right)}{r_{a}} y_{-\kappa m}\left(\vartheta_{a}, \varphi_{a}\right)
\end{array}\right),
$$

where

- the spherical coordinate system is centered at the site of the $a$ th atom,

- $\rho$ symbolizes the radial part of the wave function, including the principal quantum number $n$ of the atomic state, 
- $\kappa$ and $m$ are the usual atomic quantum numbers,

$$
\kappa= \begin{cases}-(j+1 / 2)=-(l+1) & \text { for } j=l+1 / 2 \\ j+1 / 2=l & \text { for } j=l-1 / 2\end{cases}
$$

The functions

$$
\begin{aligned}
\mathscr{y}_{\kappa m}(\vartheta, \varphi) & :=\mathscr{y}_{l j m}(\vartheta, \varphi) \\
& =\sum_{m_{s}}\left\langle l m-m_{\mathrm{s}} 1 / 2 m_{\mathrm{s}} \mid j m\right\rangle Y_{l, m-m_{s}}(\vartheta, \varphi)\left|1 / 2 m_{\mathrm{s}}\right\rangle
\end{aligned}
$$

are the spin spherical harmonics [8,9].

As a consequence of the basis expansion, the Dirac equation takes the form of a matrix equation.

The next step consists in a symmetry-dependent linear transformation of the atomic basis set. The atomic states span a space invariant under the full rotation group and under spatial inversion. By means of the basis transformation, this space is divided into several subspaces invariant under the point symmetry group of the molecule. The vectors of each subspace transform according to an irreducible representation of this group [10].

Let $D^{(i)}$ denote the $i$ th of the inequivalent irreducible representations of the molecular symmetry group. Here, the basis systems of the subspaces corresponding to $D^{(i)}$ are called

$$
\left\{|\tau i \mu\rangle, \mu=1, \ldots, n_{i}\right\}
$$

where

$\mu \quad$ counts the basis functions $|\tau i \mu\rangle$,

$n_{i} \quad$ is the dimensionality of $D^{(i)}$, and

$\tau \quad$ is an index distinguishing between different invariant subspaces belonging to the same irreducible representation $D^{(i)}$.

The elements of the group are the symmetry operations $\hat{S}$. Their effect on a symmetry orbital $|\tau i \mu\rangle$ is given by

$$
\hat{S}|\tau i \mu\rangle=\sum_{\nu=1}^{n_{i}} D_{\nu \mu}^{(i)}(S)|\tau i \nu\rangle
$$

For the symmetry-adapted basis transformation, the transformation behaviour of the atomic states under the influence of the molecular symmetry group has to be known. Application of $\hat{S}$ to a Pauli two-spinor

$$
|\rho \operatorname{aljm}\rangle:=\rho_{n l j}\left(r_{a}\right)_{y_{l j m}}\left(\vartheta_{a}, \varphi_{a}\right)
$$

with the radial function $\rho_{n l j}$ yields $[2,8]$ :

$$
\hat{S} \mid \rho \text { aljm }\rangle=(-1)^{l \tau_{s}} \sum_{m^{\prime}} R_{m}^{(j)}(\alpha \beta \gamma)\left|\rho \hat{S a l j m^{\prime}}\right\rangle
$$

Here, $\hat{S a}$ denotes the new site of the $a$ th atom, $\alpha, \beta$ and $\gamma$ are the Euler angles of the operation $\hat{S}$, and $\tau_{S}= \begin{cases}1 & \text { if } \hat{S} \text { contains a space inversion, } \\ 0 & \text { otherwise. }\end{cases}$ 
$R^{(j)}$ is the representation matrix of the full rotation group [2,8-10], given by the Wigner formula

$$
\begin{aligned}
R_{m^{\prime} m}^{(j)}(\alpha \beta \gamma)= & \mathrm{e}^{-\mathrm{i} m^{\prime} \alpha} \mathrm{e}^{-i m \gamma} \sum_{k} \frac{(-1)^{k} \sqrt{(j+m) !(j-m) !\left(j+m^{\prime}\right) !\left(j-m^{\prime}\right) !}}{k !(j+m-k) !\left(j-m^{\prime}-k\right) !\left(k+m^{\prime}-m\right) !} \\
& \times(\cos \beta / 2)^{2 j-2 k-m^{\prime}+m}(-\sin \beta / 2)^{2 k+m^{\prime}-m} .
\end{aligned}
$$

Eq. (2.4) remains valid for Dirac four-spinors if $l$ is taken to be the $l$ quantum number of the upper component [8]. A symmetry orbital

$$
|\tau i \mu\rangle=|\rho a l j m \nu i \mu\rangle
$$

is generated from an atomic state $\mid \rho$ aljm $\rangle$ by a projection operator $\hat{P}_{\mu \nu}^{(i)}[2,8]$ :

$$
\begin{aligned}
& \left.\left.\hat{P}_{\mu \nu}^{(i)} \mid \rho \text { aljm }\right\rangle=\frac{n_{i}}{h} \sum_{S} D_{\mu \nu}^{(i) *}(S) \hat{S} \mid \rho \text { aljm }\right\rangle \\
& =:|\rho a l j m \nu i \mu\rangle \\
& =:|\tau i \mu\rangle \text {, } \\
& \tau:=(\rho \text { aljm } \nu) .
\end{aligned}
$$

Here, $h$ is the order of the symmetry group.

In the case of half-integer angular momentum $j$, each spatial transformation $\hat{S}$ corresponds to two group operations $\hat{S}$ and $\bar{S}$ differing in a rotation through $2 \pi[11,12]$. Acting on a wave function with half-integer $j$,

$$
\hat{\hat{S}}=-\hat{S} \text {. }
$$

The group consisting of all operations $\hat{S}$ and $\hat{\bar{S}}$ is called a double group. In eq. $(2.6), D^{(i)}$ refers to only those irreducible representations of the double group with [8]

$$
D^{(i)}(\bar{S})=-D^{(i)}(S)
$$

As before, $h$ is the order of the single group containing the operations $\hat{S}$ only.

The explicit form of the symmetry orbitals, expressed in terms of atomic states, is given by

$$
|\rho a l j m v i \mu\rangle=\sum_{a^{\prime} m^{\prime}} C_{a m, a^{\prime} m^{\prime}}^{l j v i \mu}\left|\rho a^{\prime} l j m^{\prime}\right\rangle
$$

with the symmetry coefficients [8]

$$
C_{a m, a^{\prime} m^{\prime}}^{l j v i \mu}:=\sum_{S} \delta_{a^{\prime}, \hat{S} a} D_{\mu \nu}^{(i) *}(S)(-1)^{1 \tau_{S}} R_{m m}^{(j)}(\alpha \beta \gamma)
$$

\subsection{Linear independence of symmetry orbitals}

In general, the number of functions generated by the projection operator formalism exceeds the dimensionality of the space spanned by the atomic orbitals. Consider a set of $A$ equivalent atoms $a=1, \ldots, A$, i.e. of atoms transformed among each other by the symmetry operations. The number of states

$$
\text { | } \rho \text { aljm }\rangle
$$

with fixed quantum numbers $l$ and $j$ is then

$$
A(2 j+1) \text {, }
$$


whereas the application of the different possible projection operators,

$$
\left.\hat{P}_{\mu \nu}^{(i)} \mid \rho \text { aljm }\right\rangle=|\rho a l j m \nu i \mu\rangle,
$$

leads to $A h(2 j+1)$ functions [8]. However, it is sufficient to apply the projectors to the states of a single atom with a fixed index $a$ so that the degrees of freedom are reduced to $h(2 j+1)$.

If $A=h$, the symmetry orbitals obtained in this way are linearly independent [8].

If $A<h$, a set of linearly independent symmetry orbitals is derived with the following algorithm [8]:

1. The number $N_{i}$ of linearly independent basis systems $\left\{|\tau i \mu\rangle, \mu=1, \ldots, n_{i}\right\}$ of $D^{(t)}$ is given by

$$
N_{i}=\frac{1}{h} \sum_{S} \chi^{(i) *}(S) \sum_{a^{\prime} m^{\prime}}(-1)^{l \tau_{S}} R_{m m}^{(j)}(S) \delta_{a^{\prime}, \hat{s}_{a^{\prime}}},
$$

where $\chi^{(i)}$ denotes the characters of $D^{(i)}$.

2. For each irreducible representation $D^{(i)}, N_{i}$ linearly independent basis functions

$$
|\rho a l j m \nu i \mu\rangle=:|\tau i \mu\rangle, \quad a, l, j, i, \mu \text { fixed, }
$$

have to be found.

3. Then the $N_{i}$ basis systems

$$
\left\{|\tau i \mu\rangle, \mu=1, \ldots, n_{i}\right\}, \quad \tau=1, \ldots, N_{i},
$$

of $D^{(i)}$ automatically fulfil the condition of linear independence.

\subsection{Block structure of the matrix equation}

An essential property of the symmetry-adapted basis functions is their orthogonality. The matrix elements of any operator $\hat{H}$ invariant under the symmetry group are reduced to [10]

$$
\langle\tau i \mu|\hat{H}| \sigma k \lambda\rangle=\delta_{i k} \delta_{\mu \lambda}\langle\tau i\|\hat{H}\| \sigma i\rangle
$$

(Wigner-Eckart theorem). As a consequence, the Hamiltonian and the overlap matrix of the symmetry orbitals fall into smaller blocks along the mean diagonal. All matrix elements $\langle\tau i \mu|\hat{H}| \sigma i \mu\rangle$ with fixed values of $i$ and $\mu$ form a block. The $n_{i}$ blocks with $\mu=1, \ldots, n_{i}$ corresponding to the same irreducible representation $D^{(i)}$ are equal. Therefore, the number of matrix elements to be computed in molecular calculations is considerably reduced.

\subsection{Time reversal}

A further simplification of quasi-relativistic molecular calculations is obtained by including time reversal as an additional symmetry operation.

The time reversal operator for two-spinors is given by [10]

$$
\hat{T}=-\mathrm{i} \hat{\sigma}_{y} \hat{K}
$$

with the Pauli matrix $\hat{\sigma}_{y}$ and the operator $\hat{K}$ of complex conjugation in configuration space. The effect of $\hat{T}$ on a Pauli spinor is [8]

$$
\left.\hat{T} \mid \rho \text { aljm }\rangle=-(-1)^{l--\jmath+m} \mid \rho^{*} \text { alj }-m\right\rangle,
$$

where the radial part of the wave function is transformed into its complex conjugate. In the following, $\rho$ is 
assumed to be real so that the space of the atomic orbitals is mapped on itself by time reversal. In the case of Dirac four-spinors, this is not possible because, due to the imaginary factor $i$, the upper and the lower component show a different transformation behaviour [8]. Therefore, time reversal, as it is applied here, provides an additional simplification in the case of Pauli spinors only.

For half-integer angular momentum $j$, the matrix elements of any two states $|\psi\rangle,|\varphi\rangle$ satisfy the relations [13]

$$
\langle\psi|\hat{H}| \hat{T} \varphi\rangle=-\langle\hat{T} \psi|\hat{H}| \varphi\rangle^{*}, \quad\langle\hat{T} \psi|\hat{H}| \hat{T} \varphi\rangle=\langle\psi|\hat{H}| \varphi\rangle^{*}
$$

for an operator $\hat{H}$ invariant under time reversal.

The most important property of time reversal to be considered here is the effect on symmetry orbitals. A basis system $\left\{|\tau i \mu\rangle, \mu=1, \ldots, n_{i}\right\}$ of $D^{(i)}$ is converted into a basis set $\left\{\hat{T}|\tau i \mu\rangle, \mu=1, \ldots, n_{i}\right\}$ of the complex conjugate representation $D^{(i)} *$. Three alternatives are to be distinguished here [13-16]:

a) $D^{(i)}$ and $D^{(i) *}$ are equivalent to a real representation. Therefore, $D^{(i)}$ can be assumed to be real. The basis systems $\{|\tau i \mu\rangle\}$ and $\{|\bar{\tau} i \mu\rangle\}$ of $D^{(i)}$, given by

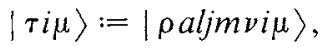

$$
\begin{aligned}
& |\bar{\tau} i \mu\rangle:=\hat{T}|\tau i \mu\rangle \\
& =-(-1)^{l-j+m}|\rho a l j-m \nu i \mu\rangle
\end{aligned}
$$

are linearly independent then and obey the relations:

$$
\begin{aligned}
& \langle\bar{\tau} i\|\hat{H}\| \sigma i\rangle=-\langle\tau i\|\hat{H}\| \bar{\sigma} i\rangle^{*}, \\
& \langle\bar{\tau} i\|\hat{H}\| \bar{\sigma} i\rangle=\langle\tau i\|\hat{H}\| \sigma i\rangle^{*} .
\end{aligned}
$$

b) $D^{(i)}$ and $D^{(i) *}$ are inequivalent, i.e.

$$
D^{(i) *}=: D^{(k)} \text {. }
$$

In this case, the basis systems $\{|\tau i \mu\rangle\}$ and $\{|\bar{\tau} k \mu\rangle\}$ with

$$
\begin{aligned}
& |\tau i \mu\rangle:=\mid \rho \text { aljmvi } \mu\rangle, \\
& |\bar{\tau} k \mu\rangle:=\hat{T}|\tau i \mu\rangle \\
& \left.=-(-1)^{1-j+m} \mid \rho \text { alj }-m \nu k \mu\right\rangle
\end{aligned}
$$

span orthogonal subspaces, and the matrix elements belonging to $D^{(i)}$ and $D^{(k)}$ are complex conjugate:

$$
\langle\bar{\tau} k\|\hat{H}\| \bar{\sigma} k\rangle=\langle\tau i\|\hat{H}\| \sigma i\rangle^{*} .
$$

c) $D^{(i)}$ and $D^{(i) *}$ are equivalent, i.e.

$$
D^{(i) *}(S)=\beta^{-1} D^{(i)}(S) \beta
$$

with a matrix $\beta$, but cannot be transformed into real form. According to Wigner [14], $D^{(i)}$ can then be chosen in such a way that $\beta$ takes the form

$$
\beta_{\mu \lambda}=\mathrm{i} f_{\lambda}^{(i)} \delta_{\bar{\mu} \lambda}
$$

with

$$
\bar{\mu}:=n_{i}-\mu+1, \quad f_{\lambda}^{(i)}:= \pm 1, \quad f_{\lambda}^{(i)}=-f_{\lambda}^{(i)} .
$$


In the following, let $\hat{S}^{\prime}$ be a symmetry operation with the Euler angles $\alpha^{\prime}, \beta^{\prime}, \gamma^{\prime}$ and $\beta^{\prime}=\pi$.

Such an operation is contained in any finite double point group possessing irreducible representations of case c). Inspection of the representation matrices published by Pyykkö and Toivonen [3] shows that for molecules with a principal axis of symmetry or with cubic symmetry, these operations can be represented by matrices of the form

$$
D_{\mu \nu}^{(i)}\left(S^{\prime}\right)=\varphi_{\nu}^{(i)} f_{\nu}^{(i)} \delta_{\bar{\mu} \nu}
$$

with a complex phase factor $\varphi_{\nu}^{(i)}$.

$D^{(i)}$ is now assumed to fulfil these requirements. We then arrive at the following results [8]:

$\left.c_{1}\right) \hat{S}^{\prime} a \neq a$ for all equivalent atoms $a$ and all symmetry operations $\hat{S}^{\prime}$ with $\beta^{\prime}=\pi$.

In this case, the basis systems of direct and reversed time are linearly independent:

$$
\hat{T}|\tau i \mu\rangle=f_{\mu}^{(i)}|\bar{\tau} i \bar{\mu}\rangle
$$

with

$$
\begin{aligned}
& |\tau i \mu\rangle:=\mid \rho \text { aljmvi } \mu\rangle, \\
& \left.|\bar{\tau} i \mu\rangle:=\eta_{\tau}^{(i)} \mid \rho \hat{S}^{\prime} \text { aljmvi } \mu\right\rangle,
\end{aligned}
$$

and the phase factor

$$
\eta_{\tau}^{(i)}:=\varphi_{\nu}^{(i)}(-1)^{l\left(1-\tau_{\mathcal{S}^{\prime}}\right)} \mathrm{e}^{\mathrm{i}\left(\gamma^{\prime}-\alpha^{\prime}\right) m}
$$

From this, the following relations are deduced:

$$
\langle\bar{\tau} i\|\hat{H}\| \bar{\sigma} i\rangle=\langle\tau i\|\hat{H}\| \sigma i\rangle^{*}, \quad\langle\bar{\tau} i\|\hat{H}\| \sigma i\rangle=\langle\tau i\|\hat{H}\| \bar{\sigma} i\rangle^{*} .
$$

$\left.c_{2}\right) \hat{S}^{\prime} a=a$ for an atom $a$ and a symmetry operation $\hat{S}^{\prime}$ with $\beta^{\prime}=\pi$.

The basis systems of direct and reversed time are linearly dependent:

$$
\hat{T}\left|\tau^{\prime} i \mu\right\rangle=f_{\mu}^{(i)} \eta_{\tau^{\prime}}^{(i)}\left|\tau^{\prime} i \bar{\mu}\right\rangle
$$

with

$$
\left|\tau^{\prime} i \mu\right\rangle:=|\rho a l j m \nu i \mu\rangle
$$

and the matrix elements

$$
\left\langle\tau^{\prime} i\|\hat{H}\| \sigma^{\prime} i\right\rangle=\eta_{\tau^{\prime}}^{(i)} \eta_{\sigma}^{(i) *\left\langle\tau^{\prime} i\|\hat{H}\| \sigma^{\prime} i\right\rangle^{*} .}
$$

The matrix elements coupling symmetry orbitals of both cases, $c_{1}$ ) and $c_{2}$ ), are

$$
\begin{aligned}
& \left\langle\bar{\tau} i\|\hat{H}\| \sigma^{\prime} i\right\rangle=\eta_{\sigma}^{(i) *}\left\langle\tau i\|\hat{H}\| \sigma^{\prime} i\right\rangle^{*}, \\
& \left\langle\tau^{\prime} i\|\hat{H}\| \bar{\sigma} i\right\rangle=\eta_{\tau}^{(i)}\left\langle\tau^{\prime} i\|\hat{H}\| \sigma i\right\rangle^{*} .
\end{aligned}
$$

In any of the three cases a), b) and c), the information needed for the calculation of matrix elements is reduced by half. A schematic sketch of the structure within the blocks, illustrating the relations between the matrix elements, is given in fig. 1 for the cases a), b) and c).

The following test, originally published by Frobenius and Schur, allows an easy classification of a given irreducible representation $D^{(i)}[15]$ :

$$
\frac{1}{h} \sum_{S} \chi^{(i)}\left(S^{2}\right)=\left\{\begin{aligned}
1 & \Rightarrow \text { case } \mathrm{a}), \\
0 & \Rightarrow \text { case } \mathrm{b}), \\
-1 & \Rightarrow \text { case } \mathrm{c}) .
\end{aligned}\right.
$$




\section{Structure of the program}

\subsection{Application}

The program computes the coefficients of a complete set of linearly independent relativistic symmetry orbitals according to eq. (2.9). The application of the routine is restricted to finite groups, i.e. to non-linear molecules. The data of the following 36 double point groups are included in the program:

$\mathrm{C}_{1}, \mathrm{C}_{\mathrm{i}}, \mathrm{C}_{2}, \mathrm{C}_{1 \mathrm{~h}}, \mathrm{C}_{2 \mathrm{~h}}, \mathrm{C}_{2 \mathrm{v}}, \mathrm{D}_{2}, \mathrm{D}_{2 \mathrm{~h}}, \mathrm{C}_{4}, \mathrm{~S}_{4}, \mathrm{C}_{4 \mathrm{~h}}, \mathrm{C}_{4 \mathrm{v}}, \mathrm{D}_{4}, \mathrm{D}_{2 \mathrm{~d}}, \mathrm{D}_{4 \mathrm{~h}}, \mathrm{C}_{3}, \mathrm{C}_{3 \mathrm{i}}, \mathrm{C}_{3 \mathrm{v}}, \mathrm{D}_{3}, \mathrm{D}_{3 \mathrm{~d}}, \mathrm{C}_{6}, \mathrm{C}_{3 \mathrm{~h}}, \mathrm{C}_{6 \mathrm{~h}}, \mathrm{C}_{6 \mathrm{v}}$, $\mathrm{D}_{6}, \mathrm{D}_{3 \mathrm{~h}}, \mathrm{D}_{6 \mathrm{~h}}, \mathrm{~T}, \mathrm{~T}_{\mathrm{h}}, \mathrm{T}_{\mathrm{d}}, \mathrm{O}, \mathrm{O}_{\mathrm{h}}, \mathrm{C}_{5 \mathrm{v}}, \mathrm{D}_{5}, \mathrm{D}_{5 \mathrm{~d}}, \mathrm{D}_{5 \mathrm{~h}}$.

\subsection{Requirements}

The structure of the irreducible representations used in the program is subject to the following requirements:

1. In case a), $D^{(i)}$ is chosen real.

2. Irreducible representations belonging to case b) have to be given in pairs of complex conjugate representations. These are stored in the program consecutively.

3. In case $c), D^{(i)}$ has to obey the conditions (2.20) and (2.21).

4. One two-dimensional or two one-dimensional irreducible representations of the group form a representation with the structure

$$
R^{(1 / 2)}(\alpha \beta \gamma)=\left(\begin{array}{cc}
\mathrm{e}^{-\mathrm{i} \alpha / 2} \cos \beta / 2 \mathrm{e}^{-\mathrm{i} \gamma / 2} & -\mathrm{e}^{-\mathrm{i} \alpha / 2} \sin \beta / 2 \mathrm{e}^{\mathrm{i} \gamma / 2} \\
\mathrm{e}^{\mathrm{i} \alpha / 2} \sin \beta / 2 \mathrm{e}^{-\mathrm{i} \gamma / 2} & \mathrm{e}^{\mathrm{i} \alpha / 2} \cos \beta / 2 \mathrm{e}^{\mathrm{i} \gamma / 2}
\end{array}\right)
$$

\subsection{Survey of the program structure}

The subroutine TSYM forms the main part of the program. This routine receives the input data from the calling program and calls several smaller subroutines executing special parts of the calculation. Specific group data are contained in a further set of subroutines and in BLOCK DATA.

a)

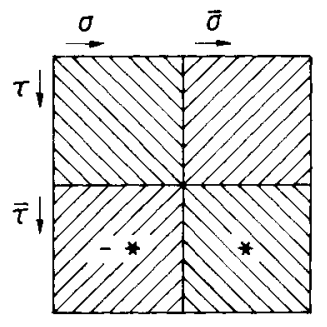

b)

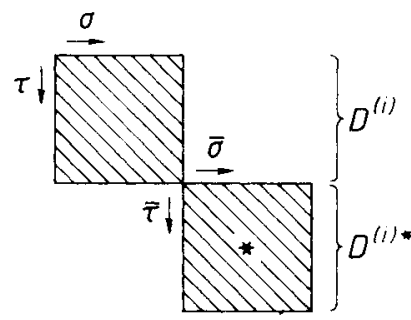

c)

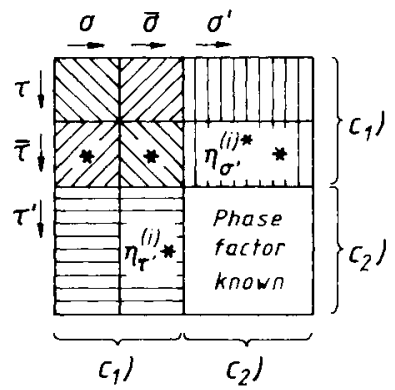

Fig. 1. Block structure in case a), b) and c). Sectors with the same hatching are related by complex conjugation (*) and, in some cases, an additional phase factor. 
In the following, the characteristic features of the program are outlined:

1. Input of atomic data

The input mainly consists of the name of the symmetry group and the coordinates and quantum numbers of the atoms, as described in detail in section 4 . The atoms are divided into sets of equivalent atoms. For each set, the input may be restricted to the data of one single atom. The coordinates of the remaining atoms are reconstructed by the program.

2. Calculation of Euler angles

The Euler angles are computed according to the method of Slater [17]:

Any point symmetry operation can be separated into a rotation $\hat{R}$ and, in some cases, a spatial inversion. The effect of $\hat{R}$ on a wave function $\psi$ is given by

$$
\hat{R} \psi(\boldsymbol{x})=\psi\left(\hat{R}^{-1} \boldsymbol{x}\right)=: \psi(A \boldsymbol{x}),
$$

where $A$ is a matrix. The Euler angles $\alpha, \beta$ and $\gamma$, as defined by Edmonds [9], are deduced as follows:

$$
\text { If } \begin{aligned}
A_{33}^{2}=1 & & & \\
& \sin \alpha= \pm A_{32} / K, & & \cos \alpha= \pm A_{31} / K, \\
& \sin \beta= \pm K, & & \cos \beta=A_{33}, \\
& \sin \gamma= \pm A_{23} / K, & & \cos \gamma=\mp A_{13} / K,
\end{aligned}
$$

with $K:=\sqrt{1-A_{33}^{2}}$.

Either the upper or the lower signs are to be used together. In the program, the upper signs are used.

$$
\text { If } \begin{aligned}
A_{33}= & +1: \\
& \cos \beta=1, \\
& \cos (\gamma+\alpha)=A_{11}=A_{22}, \\
& \sin (\gamma+\alpha)=A_{12}=-A_{21} .
\end{aligned}
$$

If $A_{33}=-1$ :

$$
\begin{aligned}
& \cos \beta=-1, \\
& \cos (\gamma-\alpha)=-A_{11}=A_{22}, \\
& \sin (\gamma-\alpha)=A_{12}=A_{21} .
\end{aligned}
$$

In this way, the Euler angles are derived up to a multiple of $2 \pi$. For an exact determination of their values, requirements 4 of section 3.2 is needed. The matrices $R^{(1 / 2)}(\alpha \beta \gamma)$ are calculated and adjusted to the given irreducible representations of the group by means of a suitable choice of $\alpha, \beta$ and $\gamma$.

3. Construction of symmetry orbitals

For each set of equivalent atoms, the symmetry orbitals are constructed by applying the projectors to the states of one single atom. In case $c_{2}$ ), this atom is mapped on itself by a symmetry operation $\hat{S}^{\prime}$ with $\beta^{\prime}=\pi$ (see section 2.4). Otherwise, the first atom of the set is chosen.

Linearly independent symmetry orbitals are selected by means of the algorithm described in section 2.2 and under consideration of time reversal. To verify the linear independence of a given set of basis functions, the determinant of their overlap matrix is calculated. The symmetry orbitals are computed in an order ensuring that the block structure is simplified as far as possible. In the case of Pauli spinors, the matrix elements within the blocks show the structure displayed in fig. 1. 


\subsection{List of subroutines of the program TSYM}

INVERS computes the transformation matrices of the atomic coordinates. Moreover, the inverse symmetry operations and the spherical coordinates of the transformed axes are calculated. These data are needed for the determination of the Euler angles.

ROAT computes $\hat{S a}$ for a given atom $a$ and all group operations $\hat{S}$.

FROSCH performs the Frobenius-Schur test (2.30).

EULER calculates the Euler angles and checks the existence of a space inversion.

FINDAT determines the atom whose states the projection operators are acting on. In case c), the operation $\hat{S}^{\prime}$ with $\beta^{\prime}=\pi$ is selected.

DJNMB calculates the $\beta$-dependent part of $R_{m m}^{(j)}(\alpha \beta \gamma)$ (eq. (2.5)).

FACT is called by DJNMB to compute $n$ ! for integer values of $n$.

SAMMA computes the matrix

$$
(-1)^{I^{\prime} S_{S}} R^{(j)}(\alpha \beta \gamma)
$$

using the results of DJNMB.

TIMFAC calculates the phase factor occurring in the relation between symmetry orbitals of direct and reversed time: If a relation of the form

$$
|\sigma k \lambda\rangle=\varphi \hat{T}|\tau i \mu\rangle
$$

holds, the phase factor $\varphi$ is associated with the symmetry orbital $|\sigma k \lambda\rangle$.

PROJEC calculates the symmetry coefficients

$$
C_{a m, a^{\prime} m^{\prime}}^{l j p i \mu}
$$

for variable values of $a^{\prime}$ and $m^{\prime}$ (eq. (2.9)).

TIMREV uses the results of PROJEC to derive the symmetry coefficients corresponding to reversed time in the cases a) and b).

INDEP verifies the linear independence of symmetry orbitals.

OUTPUT controls the output. For details, see section 5.

The following subroutines contain the matrices of the irreducible representations and other specific data of the groups:

Subroutine: Groups:

$\begin{array}{ll}\text { BASC1 } & \mathrm{C}_{1}, \mathrm{C}_{\mathrm{i}} \\ \text { BASC2 } & \mathrm{C}_{1 \mathrm{~h}}, \mathrm{C}_{2}, \mathrm{C}_{2 \mathrm{~h}} \\ \text { BASD2 } & \mathrm{C}_{2 \mathrm{v}}, \mathrm{D}_{2}, \mathrm{D}_{2 \mathrm{~h}} \\ \text { BASC4 } & \mathrm{C}_{4}, \mathrm{~S}_{4}, \mathrm{C}_{4 \mathrm{~h}} \\ \text { BASD4 } & \mathrm{C}_{4 \mathrm{v}}, \mathrm{D}_{4}, \mathrm{D}_{2 \mathrm{~d}}, \mathrm{D}_{4 \mathrm{~h}} \\ \text { BASC3 } & \mathrm{C}_{3}, \mathrm{C}_{3 \mathrm{i}} \\ \text { BASD3 } & \mathrm{C}_{3 \mathrm{v}}, \mathrm{D}_{3}, \mathrm{D}_{3 \mathrm{~d}} \\ \text { BASC6 } & \mathrm{C}_{6}, \mathrm{C}_{3 \mathrm{~h}}, \mathrm{C}_{6 \mathrm{~h}} \\ \text { BASD6 } & \mathrm{C}_{6 \mathrm{v}}, \mathrm{D}_{6}, \mathrm{D}_{3 \mathrm{~h}}, \mathrm{D}_{6 \mathrm{~h}} \\ \text { BAST } & \mathrm{T}, \mathrm{T}_{\mathrm{h}} \\ \text { BASO } & \mathrm{T}_{\mathrm{d}}, \mathrm{O}, \mathrm{O}_{\mathrm{h}} \\ \text { BASD5 } & \mathrm{C}_{5 \mathrm{v}}, \mathrm{D}_{5}, \mathrm{D}_{5 \mathrm{~d}} \\ \text { BASD5H } & \mathrm{D}_{5 \mathrm{~h}}\end{array}$


Finally, the information on the transformation behaviour of the coordinate axes is stored in BLOCK DATA for all 36 groups.

\subsection{Contents of COMMON blocks}

INPUT Input data transferred by the calling program

ATRANS Transformation properties of atomic sites ( $\hat{S a}$ for all atoms $a$ and all operations $\hat{S}$ )

EQUIVA Data concerning the sets of equivalent atoms

OPERAT Order of the group and spherical coordinates of the transformed axes as taken from BLOCK DATA

SPHERI Transformation matrices of atomic coordinates and data needed for the calculation of Euler angles

ANGLES Euler angles and indicator of space inversion

IRREPS Number of irreducible representations of the given group; names and dimensionalities of these representations

MATRIX Matrices of the irreducible representations of the given group

SPIN: Indicator of the irreducible representations acting on the eigenfunctions of $\operatorname{spin} 1 / 2$

DBETA $\beta$-dependent part of the matrices $R^{(j)}(\alpha \beta \gamma)$ for the different possible values of $\beta$

TIME Results of the Frobenius-Schur test (2.30)

CTIME Indicators needed for the construction of the basis set corresponding to reversed time in case c)

BLOCKS Information on the block structure

DEGEN Data indicating the degeneracy of blocks

SYMORB Quantum numbers $a, l, j, m, \nu, i, \mu$ of symmetry orbitals

COEFF Non-zero symmetry coefficients (2.9)

Several additional COMMON blocks contain the information stored in BLOCK DATA.

A detailed description of the COMMON variables is included in the program listing.

\section{Input}

\subsection{Input data}

1. Name of the group: INTEGER GROUP (Format A4)

2. Control parameters: INTEGER IPRINT, IFILE, INDCO

Choice of output:

- Output in readable form, suitable for printing:

IPRINT $\leqslant 0$ : No output in this form

IPRINT $>0$ : Writing of output data on a file IPRINT

- Output in compact form, suitable for storing on a file:

IFILE $\leqslant 0: \quad$ No output in this form

IFILE $>0$ : Writing of output data on a file IFILE

Choice of atomic coordinates:

INDCO $=1:$ Input of Cartesian coordinates $x, y, z$

INDCO $=2:$ Input of cylindrical coordinates $r, \varphi, z$

$\mathrm{INDCO}=3:$ Input of spherical coordinates $r, \vartheta, \varphi$ 
3. Input of atomic sites and quantum numbers:

In the input, each set of equivalent atoms is represented by one atom. If the input contains the data of several equivalent atoms, the program adjusts them to the proper set, provided that their orbitals are identical. Otherwise, an error message appears. The input consists of several data sets, each containing the coordinates of one atom and the quantum numbers $l$ and $2 j$ of its states.

Number of data sets: INTEGER NDAT

For each data set IDAT $=1$, NDAT:

Coordinates of one atom

(Angles in degrees, $-180^{\circ}<\varphi \leqslant 180^{\circ}$ ): $\quad \operatorname{REAL} * 8 \operatorname{CODAT}(\mathrm{K}, \mathrm{IDAT}), \mathrm{K}=1,3$

Number of atomic orbitals:

For each orbital IORB $=1$, NORBIT $($ IDAT):

Quantum number $l$ :

Quantum number $2 j$ :

INTEGER NORBIT (IDAT)

INTEGER LVAL(IORB,IDAT)

INTEGER JVAL(IORB,IDAT)

The input data are transferred by the calling program in the COMMON block

COMMON /INPUT/ CODAT (3,10), GROUP, LVAL (10,10), JVAL (10,10), NORBIT (10), NDAT, IPRINT, IFILE, INDCO.

\subsection{Choice of the coordinate system}

The orientation of the coordinate axes used in the input is dependent on the choice of the irreducible representations. Here, the following rules have to be obeyed [3]:

1. For molecules with a principal axis of symmetry (groups $\mathrm{C}_{n \ldots}, \mathrm{D}_{n}, \ldots, \mathrm{S}_{n}$ ):

- The $z$ axis coincides with the principal axis.

- If a reflection plane exists perpendicular to the principal axis, it is chosen as $x y$ plane.

-- If the molecule possesses $C_{2}$ axes perpendicular to $z$, one of them is taken as $y$ axis, except in the case of the group $\mathrm{D}_{3 \mathrm{~h}}$, where the $x$ axis is a $\mathrm{C}_{2}$ axis.

- The $x z$ plane is one of the mirror planes of $\mathrm{C}_{n \mathrm{v}}$.

2. For molecules of cubic or tetrahedral symmetry (groups $T, T_{h}, T_{d}, O, O_{h}$ ):

The axes $x, y, z$ are taken as $\mathrm{C}_{4}$ axes.

\section{Output}

\subsection{Input data}

The output begins with the name of the group, the Cartesian coordinates of the atoms, divided into sets of equivalent atoms and their quantum numbers. These data are taken from the COMMON blocks /INPUT/ (see section 4.1) and /EQUIVA/:

\section{COMMON /EQUIVA/:}

$\operatorname{ACO}(K, I A), K=1,3$ Cartesian coordinates of the atom with index IA

IATSET (ISET) Index of the first atom belonging to the set ISET of equivalent atoms

INDSET (IA) Index of the set containing the atom IA

NEQUAT (ISET) Number of atoms belonging to ISET

INDDAT (ISET) Index of the data set containing the data of ISET in the input (see section 4.1)

NSET Number of sets of equivalent atoms 


\subsection{Results}

The output data are contained in the following four COMMON blocks:

COMMON /BLOCKS/:

NAMBLO (K,IBLO) Name of the block IBLO:

$\mathrm{K}=1$ : name of the irreducible representation $D^{(i)}$

$\mathrm{K}=2$ : basis function index $\mu$

NSOBLO (IBLO) Number of symmetry orbitals belonging to the block IBLO

NBLO Total number of blocks

COMMON /DEGEN/:

MYDEG (IBLO) Degree of degeneracy of the block IBLO:

$\mu=1:$ MYDEG $($ IBLO $)=n_{i}$

$\mu>1:$ MYDEG (IBLO) $=0$

KRATYP (IBLO) Classification of the representation $D^{(i)}$ of the block IBLO:

$=\mathrm{A}:$ case $\mathrm{a}$ )

$=\mathrm{B} 1$ : case $\mathrm{b}$ )

$=\mathrm{B} 2$ : case $\mathrm{b}$ ), complex conjugate representation

$=\mathrm{C}:$ case $\mathrm{c}$ )

COMMON /SYMORB/:

This COMMON block contains the parameters characterizing the symmetry orbital with the index ISO, given by

$$
\mid \rho \text { aljmvin }\rangle \text { : }
$$

FACTOR(ISO) Phase factor as given in eq. (3.4)

IATSO(ISO) Index of atom $a$

LSO(ISO) Quantum number $l$

JSO(ISO) Quantum number $2 j$

MSO(ISO) Quantum number $m$

NYSO(ISO) Index $\nu$

IRSO(ISO) Index $i$ of the representation $D^{(i)}$

MYSO(ISO) Basis function index $\mu$

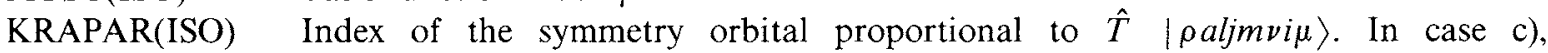
KRAPAR(ISO) $=0$.

IFCOSO(ISO) Index of the first non-vanishing symmetry coefficient of ISO (eq. (2.9))

NSO Total number of symmetry orbitals

\section{COMMON /COEFF $/$ :}

This COMMON block contains the non-zero symmetry coefficients, numbered by the index ICO (eq.

(2.9)):

$$
C_{a m, a^{\prime} m^{\prime}}^{l j v i \mu}
$$

SYMCO(ICO) Symmetry coefficient with the index ICO

IATCO(ICO) Atom index $a^{\prime}$

$\mathrm{MCO}(\mathrm{ICO}) \quad$ Quantum number $m^{\prime}$

$\mathrm{NCO}$ Total number of non-vanishing symmetry coefficients

If IPRINT $>0$, the output data are printed in self-explaining form. 


\subsection{Output on a file}

If IFILE $>0$, an output with the following structure is generated:

Name of the group:

\section{GROUP FORMAT (A4)}

Number of sets of equivalent atoms:

NSET FORMAT (I5)

For each set:

Set index, number of atoms, number of orbitals:

ISET, NEQUAT (ISET), NORBIT (IDAT) FORMAT (3I5)

(with IDAT $=$ INDDAT $($ ISET), see section 5.1)

For each atom:

Atom index, Cartesian coordinates:

IA, $(\mathrm{ACO}(\mathrm{K}, \mathrm{IA}), \mathrm{K}=1,3) \quad$ FORMAT $(5 \mathrm{X}, \mathrm{I}$, 1X,3F15.10)

For each orbital IORB:

Quantum numbers $l, 2 j$ :

LVAL (IORB,IDAT), JVAL (IORB,IDAT) FORMAT (10X,2I5)

Total number of blocks:

NBLO FORMAT (I5)

Total number of symmetry orbitals:

NSO FORMAT (I5)

Total number of non-zero symmetry coefficients:

NCO FORMAT (I5)

Degree of degeneracy of the blocks:

$(\mathrm{MYDEG}(\mathrm{IBLO}), \mathrm{IBLO}=1, \mathrm{NBLO}) \quad$ FORMAT (12I5)

Case:

$($ KRATYP (IBLO), IBLO = 1,NBLO) FORMAT $(3 \mathrm{X}, 12(1 \mathrm{X}, \mathrm{A4}))$

For each block IBLO $=1$, NBLO:

Number of symmetry orbitals, name of the block:

NSOBLO (IBLO), (NAMBLO (K,IBLO), K=1,2) FORMAT (I5,1X,A4,I2)

For each symmetry orbital ISO of this block:

Quantum numbers $l$ and $2 j$, number of symmetry coefficients, phase factor (eq. (3.4)):

LSO(ISO), JSO(ISO), NCOSO(ISO), FACTOR(ISO) FORMAT (3I5,F15.10,5X,F15.10)

For each non-zero symmetry coefficient ICO of this symmetry orbital:

Quantum number $m^{\prime}$, atom index $a^{\prime}$, symmetry coefficient:

MCO (ICO), IATCO (ICO), SYMCO (ICO) FORMAT(2I5,2F20.10)

\subsection{Error diagnostics}

In the following cases, the program stops and an error message appears, describing the corrections to be made: 


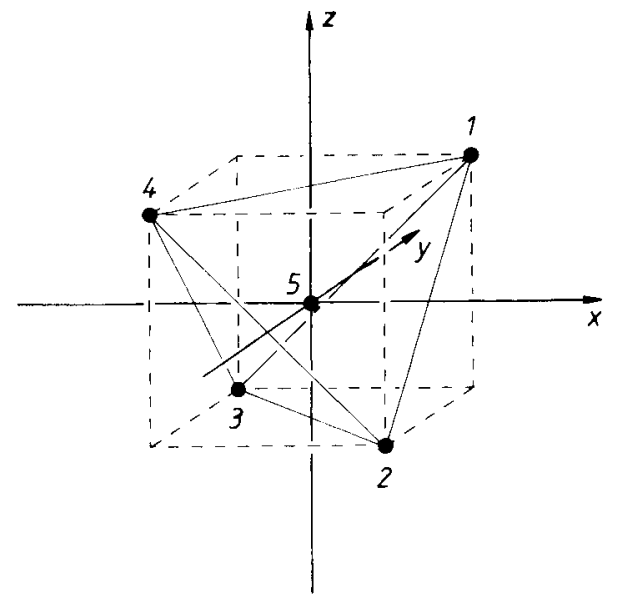

Fig. 2. Atom configuration used in the test run.

1. if the group name given in the input is not found among the groups included in the program,

2. if the control parameter INDCO (section 4.1) takes a value other than 1,2 or 3 ,

3. if the orbitals of equivalent atoms given in the input are not identical,

4. if the total number of symmetry orbitals, NSO, exceeds the dimension of the arrays of COMMON $/ \mathrm{SYMORB} /$,

5. if the total number of non-zero symmetry coefficients, NCO, exceeds the dimension of the arrays of COMMON / COEFF/.

In any of these cases, the program has to be started again with the correct data.

\section{Test run output}

In the test run, symmetry orbitals are calculated for a molecule consisting of five atoms in the configuration shown in fig. 2 . The symmetry group is $T_{d}$. Its irreducible representations are two-dimensional and belong to case c). The five atoms are divided into two sets. The first set consists of four atoms forming a tetrahedron, whereas the second set contains the central atom only. The symmetry orbitals of the molecule serve as an example of case $c_{2}$ ) (see section 2.4).

\section{References}

[1] J. Oreg and G. Malli, J. Chem. Phys. 65 (1976) 1746, 1755.

[2] A. Rosén, Intern. J. Quant. Chem. 13 (1978) 509.

[3] P. Pyykkö and H.T. Toivonen, Acta Academiae Aboensis Ser. B 43 (2) (1983).

[4] H.T. Toivonen and P. Pyykkö, Intern. J. Quant. Chem. 11 (1977) 695.

[5] D.E. Ellis and G.L. Goodman, Intern. J. Quant. Chem. 25 (1984) 185.

[6] P. Hafner, J. Phys. B 13 (1980) 3297.

[7] N. Rösch, Chem. Phys. 80 (1983) 1.

[8] J. Meyer, Intern. J. Quant. Chem. 33 (1988) 445.

[9] A.R. Edmonds, Angular Momentum in Quantum Mechanics (Princeton Univ. Press, Princeton, 1974).

[10] A. Messiah, Quantum Mechanics, vol. 2 (North-Holland, Amsterdam, 1964).

[11] V. Heine, Group Theory in Quantum Mechanics (Pergamon Press, Oxford, 1966). 
[12] G.F. Koster, Space Groups and Their Representations (Academic Press, New York, 1957).

[13] D.F. Johnston, Proc. Roy. Soc. A 243 (1958) 546.

[14] E.P. Wigner, Göttinger Nachrichten 31 (1932) 546.

[15] M. Tinkham, Group Theory and Quantum Mechanics (McGraw-Hill, New York, 1964).

[16] J.F. Cornwell, Group Theory in Physics, vol. 1 (Academic Press, London, 1984).

[17] J.C. Slater, Quantum Theory of Molecules and Solids, vol. 2 (McGraw-Hill, New York, 1965). 


\section{TEST RUN OUTPUT}

SYMMETRY ORBITALS POR THE DOUBLE GROUP TD

ATOMIC DATA:

NUMBER OF SETS OF EQUIVALENT ATCMS: 2

1. SET: NUMBER OF EOUIVALENT ATOMS: 4 NUMBER OF ORBITALS : 2

ATOM: CARTESIAN COORDINATES:

\begin{tabular}{|c|c|c|}
\hline & $Y:$ & 2: \\
\hline 1.00000000 & 1.00000000 & 1.000000000 \\
\hline & -1.00000000 & -1.00000000 \\
\hline-1.000000000 & 1.00000000 & -1.00000000 \\
\hline & 1.00000000 & 1.0000 \\
\hline
\end{tabular}

QUANTUM NUMBERS:

I: $\mathrm{J}:$

$11 / 2$

2. SET: NUMBER OF EQUIVALENT ATOMS: 1

NLMBER OF ORBITALS

ATOM: CARTESIAN COORDINATES:

$X: \quad Y: \quad \mathrm{Z}$ :

50.00000000 .000000000 .00000000

QUANTUM NUMBERS:

L: $\mathrm{J}$ :

$1 \quad 1 / 2$

$13 / 2$

SYMMETRY BLOCKS:

TOTAL NUMBER OF BLOCKS

TOTAL NUMBER OF SYMMETRY ORBITALS $\quad: \quad \begin{array}{r} \\ \text { TOLAL }\end{array}$

TOTAL NUMBER OF NON-ZERO SYMMETRY COEFFICIENTS: 206

\begin{tabular}{|c|c|c|c|}
\hline \multicolumn{2}{|c|}{ BLOCK: } & $\begin{array}{l}\text { DESREE OF } \\
\text { DESENERACY: } \\
\text { (O FOR } \\
\text { DEGENERATE } \\
\text { BLOCKS) }\end{array}$ & $\begin{array}{l}\text { TIME REVERSAL } \\
\text { CASE: } \\
\text { (B1, B2 FOR } \\
\text { COMPLEX CONJUGATE } \\
\text { RERRESENTATIONS) }\end{array}$ \\
\hline D6 & 1 & 2 & $\mathrm{c}$ \\
\hline D6 & 2 & 0 & c \\
\hline D? & 1 & 2 & c \\
\hline D7 & 2 & 0 & $\mathrm{c}$ \\
\hline 18 & 1 & 4 & c \\
\hline D6 & 2 & 0 & $\mathrm{c}$ \\
\hline D8 & 3 & 0 & C \\
\hline DB & 4 & 0 & $\mathrm{c}$ \\
\hline
\end{tabular}


SYMMETRY ORBITALS:

$\begin{array}{ll}\text { BLOCK: } & 1 \\ \text { NUMBER OF SYMMETRY ORBITALS: } & 2\end{array}$

S.O.: COBTT. :

SYMMETRY ORBITAL:
A L J M NY I MY,

$211 / 21 / 21$ D6 1$)=(-0.0833333333,0.0000000000) \quad 1 \quad 11 / 21 / 2$, $+(-0.0833333333,-0.0833333333) 111 / 2-1 / 2$

$+(0.0833333333,0.0000000000) 211 / 21 / 2)$

$+(-0.0833333333,0.0833333333) \quad 2 \quad 11 / 2-1 / 2$

$+(0.0833333333,0.000000000) \quad 3 \quad 11 / 2 \quad 1 / 2$

$+(0.0833333333,-0.0833333333) \quad 3 \quad 11 / 2-1 / 2)$

$+(-0.0833333333,0.000000000) \quad 4 \quad 11 / 2 \quad 1 / 2)$

$+(0.0833333333,0.0833333333) \quad 4 \quad 11 / 2-1 / 2)$

29

10
11
12
13
14
15
16
17
18
19
20

$213 / 2 \quad 3 / 21$ DE 1 ) =

$+(-0.0721687836,0.0721687836) 113 / 21 / 2$

$+(-0.0721687836, \quad 0.0000000000) \quad 1 \quad 13 / 2-1 / 2$,

$+(0.1250000000,0.0000000000) 213 / 2 \quad 3 / 2$

$+(0.0721687836,-0.0721687836) 2213 / 2 \quad 1 / 2$

$+(0.0000000000,0.0721687836) \quad 2 \quad 13 / 2-1 / 22$

$+(-0.1250000000, \quad 0.0000000000) 313 / 23 / 2)$

$+(0.0721687836,-0.0721687836) \quad 3 \quad 13 / 2 \quad 1 / 2$

$+(0.0000000000,-0.0721687836) 313 / 2-1 / 2$

$+(0.0000000000,0.1250000000) \quad 4 \quad 13 / 23 / 2$

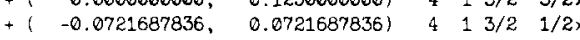

$+(0.0721687836,0.0000000000)$ \& $13 / 2-1 / 2$ s

BIOCK: D6 2

NUMBER OF SYMMETRY ORBITALS:

2

S.0.: COETF.:

SYMMETRY ORBITAL:

REAL PART: IMAGINARY PART:
ATOMIC ORBITAL:

A" L J M",

$(-1.0000000,0.0000000)$ T $211 / 21 / 21$ D6 $1,=$

21
22
23
24
25
25
27
28

$-0.0833333333, \quad 0.0833333333$

$1 \quad 1 \quad 1 / 2 \quad 1 / 2$

$+(0.0833333333,0.0000000000)$

$11 / 2-1 / 2$

$-0.0833333333,-0.0833333333)$

$211 / 2 \quad 1 / 2$

$\begin{array}{rrr}+ & 0.0833333333, & 0.0833333333 \\ +\quad-0.0833333333, & 0.0000000000\end{array}$

$21 \frac{1 / 2}{2}-1 / 2$

$\begin{array}{llll}3 & 1 & 1 / 2 & 1 / 2\end{array}$

$+(0.0833333333,-0.0833333333) \quad 4 \quad 1 \quad 1 / 2 \quad 1 / 2$

28

$(0.0000000,1.0000000)$ T $213 / 23 / 21$ D6 1 , =

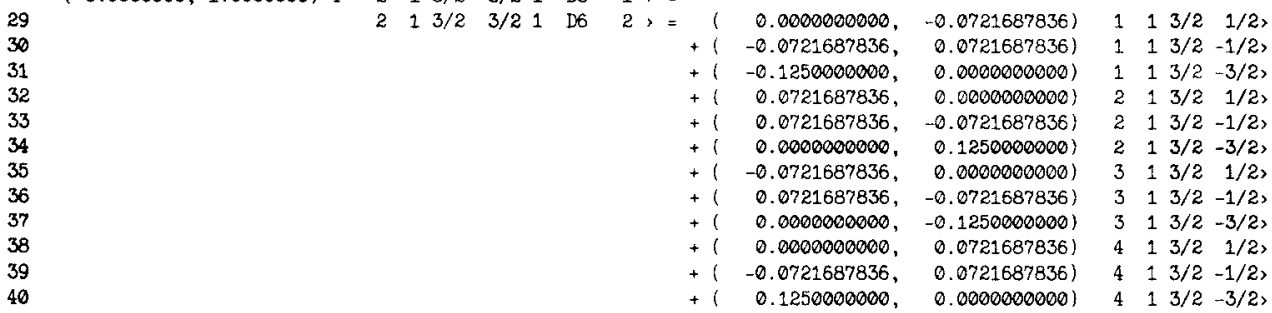


$\begin{array}{lll}\text { BLOCK: } & \text { I7 } & 1 \\ \text { NUMBER OF SYMMETRY ORBITALS: } & 3\end{array}$

S.O.: COEFT. :

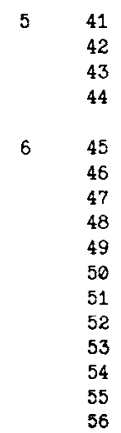

$7 \quad 57$

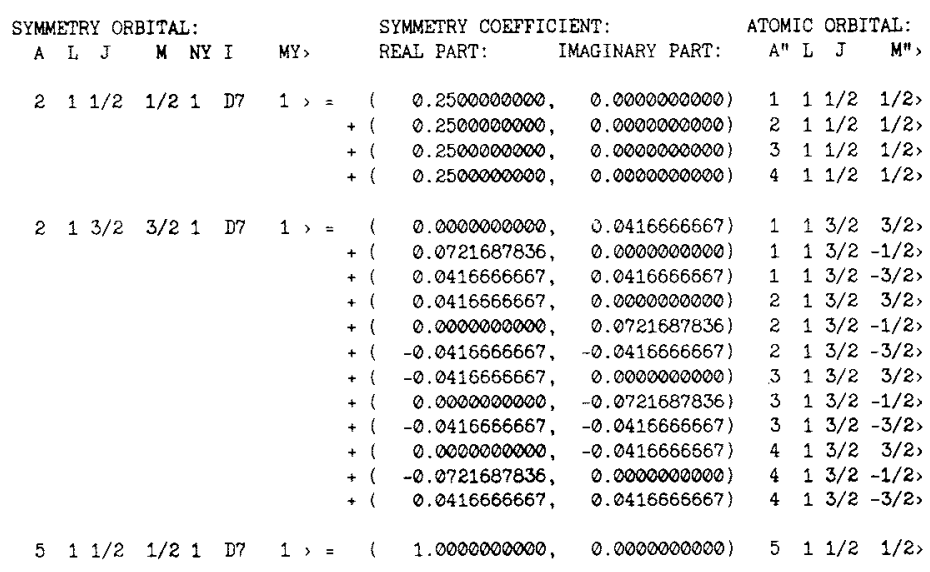

BLOCK:

DY 2
10

$(1.0000000,0.0000000) \mathrm{T} 511 / 21 / 21$ D7 1$)=$
SYMMETRY COEFFICIENT: REAL PART:

IMAGINARY PART:

ATOMIC ORBITAI:

SYMMETRY ORBITAL:
A L J M NY I MY, REAL PART: IMAGINARY PART: ANOMIC ORBITAL:

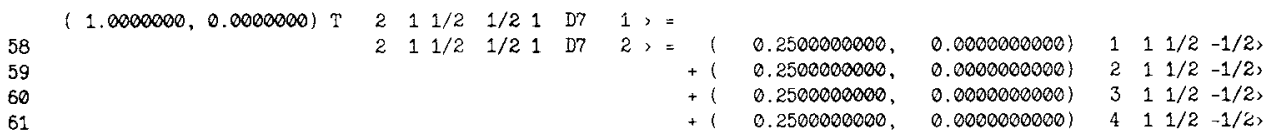

9

$(0.0000000,-1.0000000) \mathrm{T} \quad 213 / 2 \quad 3 / 21$ D7 1$)=$

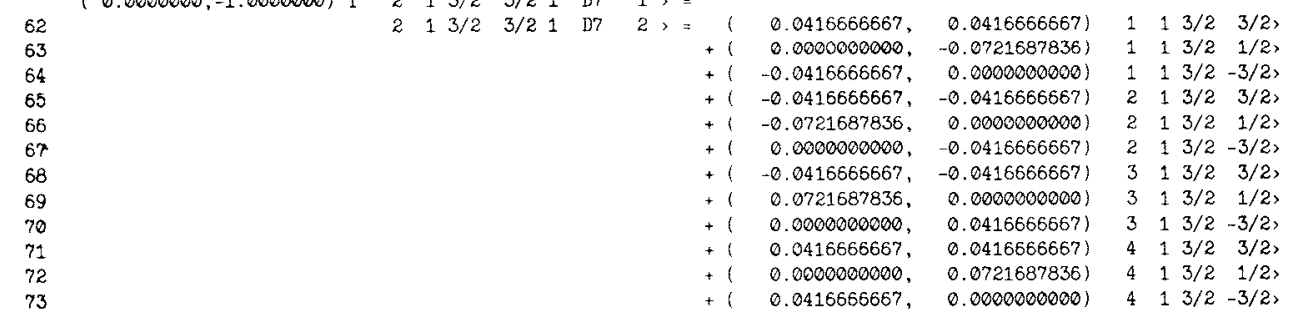
74

$511 / 21 / 21$ D7 2 , =

$1.0000000000,0.0000000000) 511 / 2-1 / 2$,

BLOCK:

DSO 1

S.O.: COEFT.:

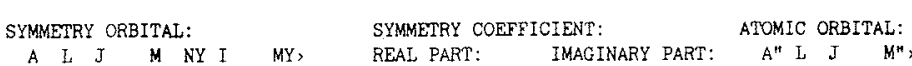

$\begin{array}{ll}11 & 75 \\ & 76 \\ & 77 \\ & 78 \\ & \\ 12 & 79 \\ & 80 \\ & 81 \\ & 82 \\ & 83 \\ & 83 \\ & 84 \\ & 85 \\ & 85 \\ & \\ & \end{array}$

$211 / 21 / 21$ n8 1 , $=(0.0000000000,0.2500000000) 111 / 21 / 2$, $+(0.2500000000,0.0000000000) 211 / 21 / 2)$

$+(-0.2500000000,0.0000000000) 311 / 21 / 2)$

$+(0.0000000000,-0.2500000000) 411 / 21 / 2)$

$213 / 23 / 21$ D8 $1,=(-0.1666666667,0.0000000000) 113 / 23 / 2\rangle$

$+(-0.0721687836,-0.0721687836) \quad 113 / 21 / 2>$

$+(-0.0415666667,0.0416665667) 113 / 2-3 / 2$ )

$+(0.1666666667,0.0000000000) 213 / 23 / 2$ )

$+(-0.0721687836,0.0721687836) 2213 / 21 / 2)$

$+(-0.0416666667,-0.0416666667) \quad 2 \quad 13 / 2-3 / 2)$

$+(0.1666666667,0.0000000000) \quad 313 / 23 / 2)$

$+(0.0721687836,-0.0721687836) 313 / 2 \quad 1 / 2$, 